\title{
GESTÃO EDUCACIONAL COM ENFOQUE EM INDICADORES DE DESEMPENHO DO BALANCED SCORECARD
}

\author{
http://dx.doi.org/10.5902/2318133863849
}

\author{
Geraldo Magela Jardim Barra ${ }^{1}$ \\ Rodrigo Oliveira da Silva ${ }^{2}$ \\ Marilia Medeiros Schocair ${ }^{3}$
}

\begin{abstract}
Resumo
O objetivo deste estudo foi propor um modelo de maturidade para gestão educacional de instituições de ensino superior com enfoque no balanced scorecard - BSC. Com base no instrumento de avaliação do sistema nacional de avaliação da educação superior - Sinaes - e no BSC foi elaborado o roadmap de gestão educacional. Este Roadmap foi avaliado por um grupo de especialistas. Pôde-se verificar que este modelo apresentou um mapa estratégico que pode funcionar como um instrumento para a orientação de esforços por melhores resultados de desempenho de unidades de ensino. A missão da unidade de ensino foi traduzida num conjunto de medidas de desempenho que servirão de alicerce para um sistema de medição e gestão estratégica. No roadmap o desempenho foi medido por quatro perspectivas balanceadas: pessoas, processos, posicionamento e performance que, por meio de um conjunto de objetivos e metas, se articularam numa relação de causa e efeito. O roadmap poderá ser utilizado na prescrição de ações estratégicas em unidades de ensino para que possam evoluir de um determinado nível de maturidade para um nível mais elevado, configurando um processo de melhoria contínua, tanto administrativa, quanto acadêmica.
\end{abstract}

Palavras-chave: gestão de processo; balanced scorecard; gestão educacional.

\section{EDUCATIONAL MANAGEMENT WITH A FOCUS ON BALANCED SCORECARD PERFORMANCE INDICATORS}

\section{Abstract}

The aim of this study is to propose a maturity model for educational management of higher education institutions focusing on the balanced scorecard - BSC. Based on the Evaluation Instrument of the National Higher Education Evaluation System - Sinaes - and the BSC, the educational management roadmap was built. This roadmap was evaluated by a group of experts. It was verified that this model presented a strategic map that can act as an instrument for guiding efforts for better performance results of teaching units. The teaching unit's mission will be translated into a set of performance measures that will underpin a strategic measurement and management system. In the Roadmap, performance will be measured within four balanced perspectives: People, Processes, Positioning, and Performance, which, through a set of objectives and goals, the performance vectors articulate into a cause and effect relationship. The roadmap can be used to prescribe strategic actions in educational units so that they can evolve from a certain level of maturity to a higher level, configuring a process of continuous administrative and academic improvement.

Key-words: process management; balanced scorecard; educational management.

\footnotetext{
1 Universidade Federal de São João Del-Rei, Brasil. E-mail: gmjbar@yahoo.com.br.

2 Universidade Federal de Juiz de Fora, Brasil. E-mail: oliveira.silva@ufjf.edu.br.

3 Universidade do Vale do Rio dos Sinos, Brasil. E-mail: marilia.schocair@gmail.com. 


\section{Introdução}

as duas décadas iniciais do século 21 notou-se crescente discussão, dentro do ambiente acadêmico, a respeito da necessidade do uso de formas de gestão educacional baseadas em desempenho, face às mudanças na sociedade moderna (Alboushra et al, 2015; Azma, 2010). De fato, novas formas de gestão educacional, baseadas em desempenho, se fazem necessárias em um ambiente cada vez mais competitivo.

Mediante o cenário de fragmentação vivido pelo setor educacional brasileiro desde os anos 2000, a proposição de um modelo de maturidade para processos de gestão educacional capaz de propiciar alternativas à gestão de instituições de ensino superior se faz necessária. Para implantar novas abordagens de avaliação de desempenho é imprescindível que se faça o mapeamento dos processos gerenciais e acadêmicos das IES, para que, dessa forma, se possa definir em qual estágio de maturidade a mesma se encontra estruturada.

Entende-se que uma gestão estratégica baseada em desempenho é fundamental para orientação do percurso acadêmico e é condição prévia para implantação de novas metodologias de gestão educacional. A medição do desempenho em IES tem atraído atenção de pesquisadores dessa área (Alach, 2017, Alboushra et al, 2015). Na área de gestão educacional as abordagens para a avaliação de desempenho têm evoluído significativamente, com destaque para o BSC. Diversos estudos vêm demostrando o emprego da abordagem BSC para a mensuração do desempenho de IES (Nayeri, 2008; Yu, 2009; Hladchenko, 2015; De Andrade, 2018).

Assim sendo, este trabalho teve como objetivo geral propor a elaboração de um modelo de maturidade para gestão educacional de IES com enfoque no BSC. Para tanto, com base no instrumento de avaliação de cursos de graduação presencial e a distância (Sinaes, 2017), e de acordo com as perspectivas do BSC, foi elaborado um modelo de maturidade para gestão educacional de IES. Este modelo de maturidade foi definido neste trabalho como Roadmap de Gestão Educacional - RGE.

Este modelo de maturidade é adequado para gestão educacional de IES e, em seu âmbito, pode ser desenvolvido de forma ampla, pois se trata de uma ferramenta estratégica que tem a capacidade de implantar a missão de Instituições de Ensino Superior que são baseadas na sustentabilidade educacional.

\section{Balanced Scorecard - BSC}

O BSC foi definido como sendo um sistema de mensuração de desempenho. Com o passar do tempo, todavia, por sua evolução conceitual, o BSC foi ampliando seu escopo e foi definido como um sistema de gestão estratégica (Kaplan; Norton, 2004).

Desenvolvido no início da década de 1990 o BSC foi definido como um meio para traduzir a missão e a estratégia de uma organização em objetivos e instrumentos para mensuração de desempenho, promovendo a comunicação, a informação e o aprendizado. De fato, o diferencial da ferramenta em relação a outros métodos de avaliação de desempenho é o seu desdobramento da missão e da estratégia da organização, não se atendo apenas a um método de controle de desempenho, mas também, um sistema de comunicação da estratégia e de propagação do aprendizado (Kaplan; Norton, 1997; Hladchenko, 2015). Pode ser, dessa forma, considerado um método abrangente para a 
avaliação do desempenho organizacional, dado que parte do estabelecimento de indicadores e metas relacionadas às diretrizes estratégicas são definidas pelos gestores (Lipe; Salterio, 2000; Bhagwat; Sharma, 2007; Dinçer et al, 2019).

Seus desenvolvedores, ao perceberem que o frequente uso de medidas financeiras baseadas em parâmetros contábeis apresentava limitações relacionadas a dimensão do tempo e de balanceamento, propuseram as perspectivas do BSC.

Para os autores os dados contábeis refletem com exatidão as ações estratégicas do passado da organização e são limitados para previsões de cenários futuros, fazendo com que tais indicadores sejam considerados inadequados a respeito do valor econômico de uma organização. Por meio da conjugação de indicadores financeiros e não financeiros, de curto e longo prazo, sintetizado em perspectivas equilibradas - financeira, clientes, processos internos, aprendizagem/crescimento -, os gestores passaram a tomar suas decisões de forma mais estruturada e racional. Essas quatro perspectivas buscam equilibrar os objetivos de curto e longo prazo, os resultados desejados e os vetores desses resultados, as medidas concretas e mais subjetivas. (Kaplan; Norton, 1997).

No BSC há a pressuposição de que os indicadores financeiros, por si só, não são suficientes para contemplar aspectos relevantes que impulsionam a rentabilidade no longo prazo. Enquanto o vetor de curto prazo é definido por medidas financeiras, os vetores de longo prazo são definidos por medidas de longo prazo (Kaplan; Norton, 1997).

Posteriormente a ferramenta teve sua área de atuação ampliada, chegando à gestão de IES, tanto públicas, quanto privadas. A medição do desempenho em IES tem atraído atenção de pesquisadores no setor educacional (Lawrence; Sharma, 2002; Cullen, 2003; Chen Et Al, 2006; Beard, 2009; Alach, 2017).

Medição do desempenho em instituições do ensino superior

Como toda e qualquer organização uma IES carece de estratégias para o alcance de seus objetivos, sejam financeiros, acadêmicos ou sociais. Assim, para determinar se seu desempenho está atingindo os objetivos, instrumentos de mensuração de desempenho devem ser empregados (Nayeri, 2008; Yu, 2009; Hladchenko, 2015; De Andrade, 2018).

A medição do desempenho em instituições de ensino superior tem atraído atenção substancial de pesquisadores (Alboushra; Shahbudin; Abdalla, 2015; Azma, 2010), muitas vezes focando na aplicabilidade e valor dos conceitos de medição de desempenho para o setor educacional (Alach, 2017).

Diversos estudos demonstram o emprego da abordagem do BSC para a mensuração do desempenho de IES, bem como mecanismos para o desdobramento da estratégia organizacional (Nayeri, 2008; Yu, 2009; Hladchenko, 2015; De Andrade, 2018).

Alach (2017) usou componentes de um modelo de maturidade de sete elementos para examinar o desenvolvimento da maturidade de medição de desempenho em universidades da Nova Zelândia no período 2008-2013.

O resultado deste estudo demonstrou que as universidades apresentaram pontos fortes no alinhamento das medidas com a direção estratégica e a melhoria no uso de estruturas de desempenho. Os resultados têm sido vistos na amplitude e qualidade das medidas e, mais importante, no uso de informações de desempenho para orientar a tomada de decisões institucionais. 
Segundo Hladchenko (2015) trata-se de uma prática comum em várias IES do mundo a elaboração de planos estratégicos que são acompanhados por um sistema de monitoramento, em especial com o uso do BSC, reconhecido como um arcabouço analítico muito relevante para o planejamento educacional.

Entretanto, Nickel (2011) destaca que é necessário adaptar o BSC para sua implantação em instituições de educação superior. Existem ativos intangíveis que permeiam as atividades dessas organizações, que necessitam transcender aos indicadores financeiros e econômicos para a constatação de seu desempenho e alcance de objetivos estratégicos. Nesse sentido Kaplan e Norton (2004) defendem que o BSC possibilita a inclusão de outras perspectivas.

Processo de medição de maturidade em processos

Os estudos de modelos de maturidade têm crescido entre acadêmicos, com aplicação em diversas áreas: projetos (PMI, 2003), cadeias de suprimentos (Lockamy; Mccormack, 2004), processos (Hammer, 2007), ensino superior (Alack, 2017), entre outros. O mesmo tem acontecido com estudos de modelos de maturidade de processos (Maull et al. 2003; Fisher, 2004; Rosemann; De Bruin, 2005; Hammer, 2007; Lee et al., 2007; Rodholff, 2009, Roglinger et. al, 2012). Dentre estes merece destaque o modelo de maturidade criado por Hammer (2007) pela sua aplicação empresarial.

No intuito de responder a demanda empresarial por gestão de processos, Hammer (2007) identificou duas características necessárias para que os processos possuam bom desempenho e o mantenha: facilitadores de processos e recursos empresariais. Surgiu então o Modelo de Processo e Maturidade Empresarial - PEMM.

Os facilitadores de processos pertencem a processos individuais e são no cinco no total: design; executante; proprietário; infraestrutura; métricas. No que diz respeito aos os recursos organizacionais quatro itens se destacam: liderança; cultura; especialização e governança. Organizações podem se utilizar dos facilitadores e dos recursos em suas avaliações de forma conjunta, visando planejar e analisar o progresso de suas transformações baseadas em processos (Hammer, 2007).

Quadro 1 -

Facilitadores e recursos da ferramenta PEMM.

\begin{tabular}{|c|l|l|}
\hline \multirow{4}{*}{$\begin{array}{c}\text { Facilitadores de } \\
\text { Processos }\end{array}$} & Design & $\begin{array}{l}\text { A abrangência da especificação de como o processo deve } \\
\text { ser executado }\end{array}$ \\
\cline { 2 - 3 } & Executante & $\begin{array}{l}\text { Executores do processo, suas habilidades e conhecimentos } \\
\text { são considerados }\end{array}$ \\
\cline { 2 - 3 } & Infraestrutura & $\begin{array}{l}\text { Executivo sênior que se responsabilize pelo processo e seus } \\
\text { trabalhos }\end{array}$ \\
\cline { 2 - 3 } & Métrica & $\begin{array}{l}\text { Medidas da organização para acompanhar o desempenho do } \\
\text { processo }\end{array}$ \\
\cline { 2 - 3 } & Liderança & Executivos seniores que apoiam a criação de processos \\
\hline
\end{tabular}




\begin{tabular}{|c|l|l|}
\hline \multirow{2}{*}{$\begin{array}{c}\text { Recursos } \\
\text { Organizacionais }\end{array}$} & Cultura & $\begin{array}{l}\text { Foco no cliente, trabalho em equipe, responsabilidade } \\
\text { pessoal e vontade de mudar }\end{array}$ \\
\cline { 2 - 3 } & Especialização & Habilidades e metodologias para o redesenho do processo \\
\cline { 2 - 3 } & Governança & $\begin{array}{l}\text { Mecanismos de gestão de projetos complexos e iniciativa de } \\
\text { mudança }\end{array}$ \\
\hline
\end{tabular}

Fonte: baseado em Hammer (2007).

No modelo de Hammer (2007) quatro níveis de intensidade - P1, P2, P3 e P4 - foram definidos para o grupo de atributos dos facilitadores de processo. Esses níveis de intensidade pressupõe uma evolução gradual em direção à melhoria dos processos, evoluindo de P1 até P4. Do mesmo modo há também, no caso das recursos organizacionais, quatro níveis de maturidade: E1, E2, E3, E4.

O modelo PEMM de Hammer (2007) apresenta uma metodologia de mudança por níveis na escala do modelo de maturidade que serviu de base teórica para o modelo deste artigo. No PEMM o progresso entre os níveis de maturidade apenas ocorrerá quando todos os cinco facilitadores de processos atingirem o mesmo nível. Quando todos os facilitadores de processos estiverem definidos no nível P1, a maturidade do processo será classificada de nível 1. Por sua vez, quando todos os facilitadores de processos estiverem avaliados em P2, o nível de maturidade do processo seria classificado como 2. Já quando todos os facilitadores de processos estiverem classificados no nível P3, a maturidade do processo será classificada como nível 3. Por fim, quando todos os facilitadores de processos estiverem avaliados em $\mathrm{P} 4$, o nível de maturidade do processo será classificado como 4.

Todavia, se no mínimo um facilitador de processos, entre os cinco, não atingir o nível $\mathrm{P} 1$, isso significará que o padrão de maturidade da organização será classificado como P0. Neste caso específico caracteriza-se o contexto no qual as organizações que não investiram na abordagem de processos. Também é necessário que todas as empresariais estejam presentes para que seja possível institucionalizar os facilitadores de processos.

É preciso que todos os recursos organizacionais estejam presentes na organização para que seja possível institucionalizar os facilitadores de processos e sustentar a performance dos processos de negócios. Do mesmo modo que observado no caso dos facilitadores de processos, há no caso dos recursos organizacionais, quatro níveis de maturidade: E1, E2, E3, E4. Para atestar o avanço em estágios ou níveis de maturidade de processos dos facilitadores de processos, somente quando todos os recursos organizacionais estiverem posicionadas em determinado nível é que se terá a condição para o avanço ao próximo estágio de maturidade. Quanto maiores forem os recursos organizacionais, maiores serão os níveis de evolução dos facilitadores de processos. No momento em que a empresa atinge o nível E1 já está preparada para avançar para o estágio P1. Quando todos os recursos organizacionais atingirem o nível E2 a empresa poderá avançar o seu nível de maturidade em processos para o estágio P2 e assim seguirá sucessivamente no avanço do modelo de maturidade. 
Por fim, a evolução entre os níveis de maturidade somente ocorre quando todos os recursos organizacionais atingirem determinado nível. Assim, no caso de todos os recursos organizacionais estarem classificadas no nível E1, a maturidade do processo será de nível 1. Caso todos os recursos organizacionais estiverem avaliadas em E2, o nível de maturidade do processo seria equivalente a 2. Quando todos os recursos organizacionais estarem classificados no nível E3, a maturidade do processo será de nível 3. Se todos os recursos organizacionais forem avaliados em E4, o nível de maturidade seria equivalente a 4 .

Com a finalidade de elaborar o RGE, que é um arcabouço analítico para aferição de maturidade da gestão educacional de uma IES, foi realizada uma pesquisa exploratória com as seguintes etapas: definição dos estágios de maturidade do modelo, construção das escalas do modelo e avaliação do modelo por especialistas. Após a definição dos estágios de maturidade do modelo e a construção das escalas, o modelo de maturidade foi avaliado por uma equipe de cinco especialistas.

\section{Roadmap para gestão educacional baseado no BSC}

A contribuição do RGE para gestão educacional está focada no modelo proposto, que permite identificar os pontos críticos de cada curso, possibilitando destacar os pontos de melhoria por meio de um roadmap, uma espécie de plano mestre para a implantação de mudanças que indica as etapas a serem tomadas em um esforço de aprimoramento gerencial.

Um roadmap é compreendido como um roteiro, cujo objetivo é estabelecer e organizar metas de desempenho. É o caminho a ser percorrido, por meio de processos aceitos e desejados, para se atingir um objetivo (Kalakota; Robinson, 2001). Para definir o RGE primeiro é importante determinar o estado de maturidade e alinhamento atuais. Depois é necessária comparação de seus níveis com aqueles que apresentaram melhores índices, respeitando os grupos pertencentes. Posteriormente a condução de um benchmarking marca o início da definição do nível da meta, e pode, também, ser um indicador de necessidade de investimento. Com base em seus resultados e na avaliação da situação atual é possível definir o nível de maturidade desejado, ou estado futuro. Depois que as situações atuais e futuras são conhecidas deve-se identificar quais questões precisam ser melhoradas. Em seguida é necessário definir quais ações são necessárias para melhorar os déficits encontrados de forma que o estado futuro seja alcançado. Depois que as ações são conhecidas é necessário definir a ordem na qual elas devem ser implantadas, ou seja, um plano mestre é traçado. Finalmente é importante que se faça reavaliações da maturidade e do alinhamento com certa frequência dentro de um ciclo de maturidade.

No RGE o desempenho da IES poderá ser medido por meio de quatro perspectivas: Pessoas, Processos, Posicionamento e Performance, articuladas em função dos objetivos, metas e vetores de desempenho derivados do planejamento estratégico da IES. Ao se optar por essa estruturação o mapa estratégico permitirá a comunicação dos objetivos estratégicos a todos os colaboradores da organização, o que contribui, em última análise, para o alinhamento estratégico dentro da organização (Kaplan; Norton, 2004). 
Com relação a medição dos processos utilizou-se as premissas do modelo de Hammer (2007). No que diz respeito à análise da relação da maturidade dos processos educacionais com o desempenho, escolheu-se aplicar as premissas de mensuração de desempenho do BSC de Kaplan e Norton (1992).

Neste estudo assume-se que o desempenho educacional deve ser avaliado por meio da combinação de diferentes indicadores balanceados. A perspectiva Pessoas foi selecionada a fim de representar os talentos humanos das unidades de ensino que sustentarão a implementação das estratégias. Para construir a perspectiva Processos foram selecionada as três dimensões do Sistema Nacional de Avaliação da Educação Superior - Sinaes (Brasil, 2017): a organização didático-pedagógica; o corpo docente; e, a infraestrutura.

O desempenho administrativo é composto pelas perspectivas de Posicionamento e Performance. A perspectiva de Posicionamento está baseada na ótica de clientes, proposta por Kaplan e Norton (1997), mas considerando as especificidades das IES. A perspectiva de Performance está alicerçada na dimensão financeira, também proposta pelos mesmos autores, entretanto, considerando a singularidade do setor educacional ligado a sustentabilidade (De Andrade et al, 2018), passando a incorporar a dimensão social. Estas duas perspectivas serão relacionadas com a perspectiva de processos educacionais: figura 1.

Figura 1 -

Perspectivas do BSC aplicadas ao Roadmap de gestão educacional.

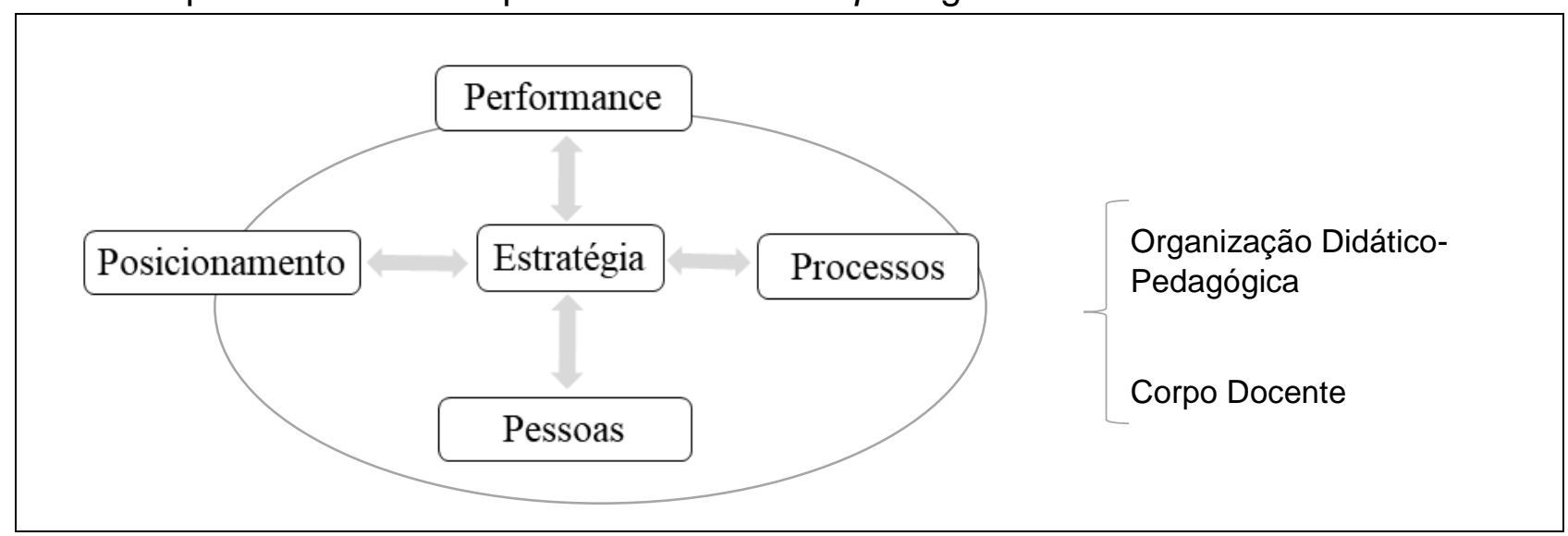

Fonte: autores.

\section{Resultados}

- Elaboração do roadmap de gestão educacional

O RGE apresenta um mapa estratégico para orientação de esforços voltados à obtenção de melhores resultados de desempenho de IES. A missão da IES será traduzida em um conjunto de indicadores que servirão de base para um sistema de gestão estratégica.

No RGE o desempenho da unidade de ensino poderá ser medido por meio das quatro perspectivas: Pessoas, Processos, Posicionamento e Performance, as quais serão articuladas em função dos objetivos, metas e vetores de desempenho derivados do planejamento estratégico da IES. Para definição do nível de maturidade das unidades 
acadêmicas foi desenvolvido um método para identificação dos turning points ou pontos de inflexão de mudança significativa. Os níveis de maturidade poderão ser mensurados por meio do somatório dos seus respectivos indicadores, utilizando a mediana como TP.

A unidade acadêmica que apresentar no RGE um escore abaixo ou igual à mediana entre dois níveis na escala de maturidade será classificada no estágio inferior de maturidade destes níveis. $O$ escore de cada unidade será calculado pelo somatório dos escores obtidos dos 52 indicadores ponderados pelos níveis de maturidade $\mathrm{M}-1, \mathrm{M}-2, \mathrm{M}$ 3, M-4, M-5, sendo M1 o nível de menor maturidade, indicado pela cor vermelha, e M5 o nível de maior maturidade, indicado pela cor verde: tabela 1).

Tabela 1 -

Turning points: maturidade acadêmica.

\begin{tabular}{c|l|c}
\hline $\begin{array}{c}\text { Nível de } \\
\text { Maturidade }\end{array}$ & \multicolumn{1}{|c|}{ Escores máximos } & $T P$ \\
\hline M1 & 52 indicadores $\times 1$ ponto = até 52 pontos & $\leq 78$ \\
\hline M2 & 52 indicadores $\times 2$ pontos = até 104 pontos & $>78 \leq 130$ \\
\hline M3 & 52 indicadores $\times 3$ pontos = até 156 pontos & $>130 \leq 182$ \\
\hline M4 & 52 indicadores $\times 4$ pontos = até 208 pontos & $>182 \leq 234$ \\
\hline M5 & 52 indicadores $\times 5$ pontos = até 260 pontos & $>234$ \\
\hline
\end{tabular}

Fonte: autores.

O escore de cada unidade acadêmica será calculado pelo somatório dos escores dos 16 indicadores das perspectivas Posicionamento e Performance ponderados pelo peso da escala de desempenho - D1, D2, D3, D4, D5 -, sendo D1 o nível de menor maturidade, indicado pela cor vermelha, e D5 o nível de maior maturidade, indicado pela cor verde: tabela 2.

Tabela 2 -

Turning points: desempenho administrativo.

\begin{tabular}{c|c|c}
\hline $\begin{array}{c}\text { Nível de } \\
\text { Desempenho }\end{array}$ & Escores máximos & TP \\
\hline D1 & 16 indicadores $\times 1$ ponto = até 16 pontos & $\leq 24$ \\
\hline D2 & 16 indicadores $\times 2$ pontos = até 32 pontos & $>24 \leq 40$ \\
\hline D3 & 16 indicadores $\times 3$ pontos = até 48 pontos & $>40 \leq 56$ \\
\hline D4 & 16 indicadores $\times 4$ pontos = até 64 pontos & $>56 \leq 72$ \\
\hline D5 & 16 indicadores $\times 5$ pontos = até 80 pontos & $>72$ \\
\hline
\end{tabular}

Fonte: autores. 
A tabela 3 apresenta um exemplo de operacionalização do radmap de gestão educacional para desempenho acadêmico. Neste exemplo uma IES hipotética está no nível de maturidade M3 com um escore de 154 pontos na perspectiva Acadêmica. Neste exemplo hipotético a definição do nível de maturidade foi realizada conforme a tabela 1 Turning points (maturidade acadêmica).

No exemplo hipotético da tabela 3 há dezoito indicadores classificados como nível 2 , dezoito indicadores classificados como nível 3 e dezesseis indicadores classificados como nível 4. Logo, o desempenho acadêmico da empresa hipotética apresenta 154 pontos, sendo classificada no nível de maturidade M3.

A tabela 4 apresenta um exemplo de operacionalização do radmap de gestão educacional em desempenho administrativo. Neste exemplo uma IES hipotética está no nível de maturidade "D3" com o escore 50 na perspectiva desempenho administrativo. Neste exemplo hipotético a definição do nível de maturidade foi realizada conforme a tabela 2 - turning points (desempenho administrativo).

No exemplo hipotético da tabela 3 há três indicadores classificados como nível 2, oito indicadores classificados como nível 3 e cinco indicadores classificados como nível 4. No exemplo hipotético da tabela 4 há dezoito indicadores classificados como nível 2, dezoito indicadores classificados como nível 3 e dezesseis indicadores classificados como nível 4. Logo, o desempenho administrativo da empresa hipotética apresenta 50 pontos, sendo classificada no nível de maturidade D3.

Tabela 3 -

Roadmap de gestão educacional no desempenho acadêmico.

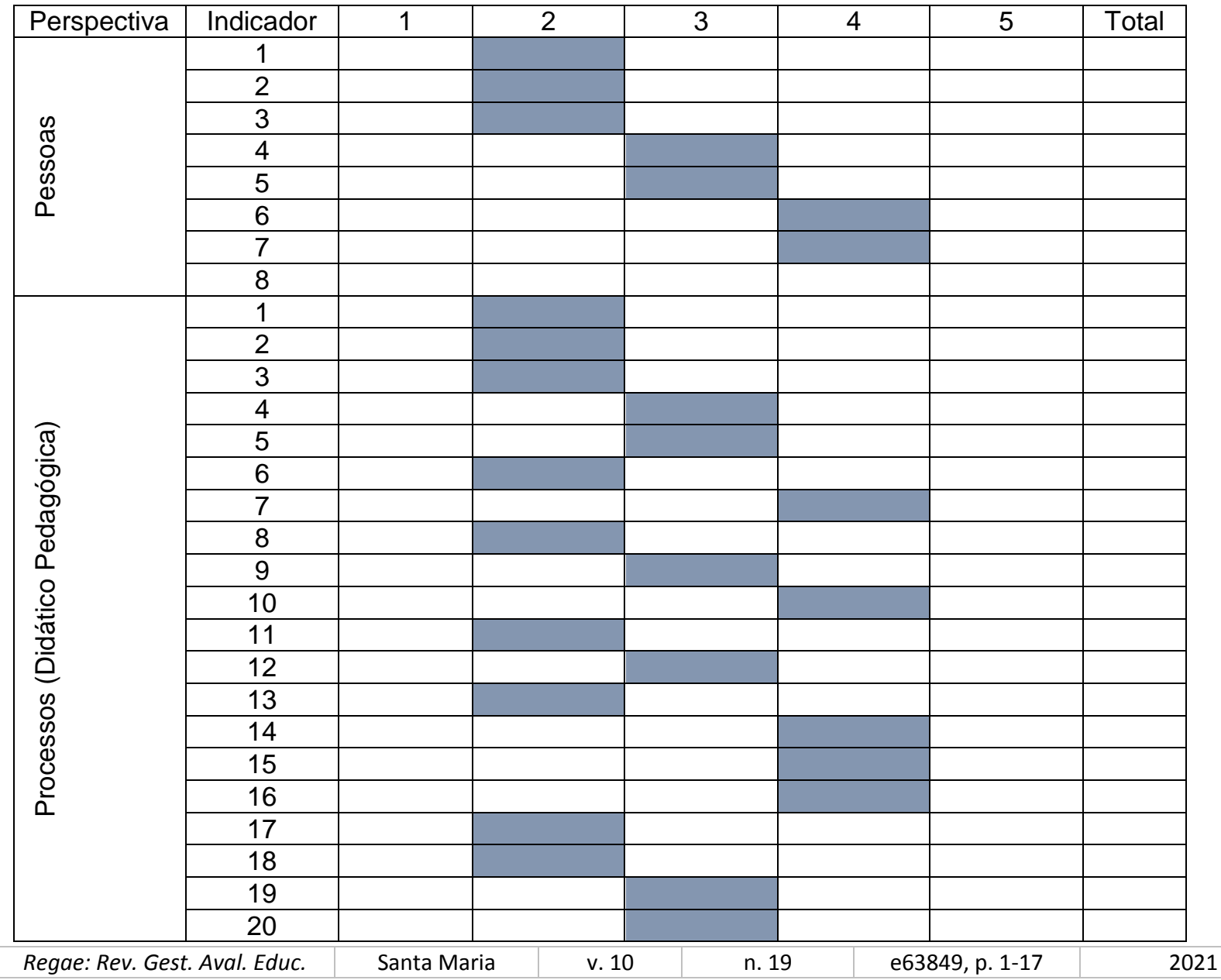




\begin{tabular}{|c|c|c|c|c|c|}
\hline \multirow{9}{*}{ 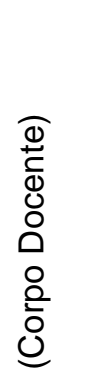 } & 1 & & & & \\
\hline & 2 & & & & \\
\hline & 3 & & & & \\
\hline & 4 & & & & \\
\hline & 5 & & & & \\
\hline & 6 & & & & \\
\hline & 7 & & & & \\
\hline & 8 & & & & \\
\hline & 9 & & & & \\
\hline \multirow{7}{*}{ 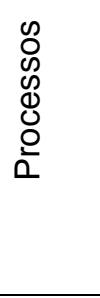 } & 10 & & & & \\
\hline & 11 & & & & \\
\hline & 12 & & & & \\
\hline & 13 & & & & \\
\hline & 14 & & & & \\
\hline & 15 & & & & \\
\hline & 16 & & & & \\
\hline \multirow{8}{*}{ 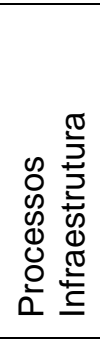 } & 1 & & & & \\
\hline & 2 & & & & \\
\hline & 3 & & & & \\
\hline & 4 & & & & \\
\hline & 5 & & & & \\
\hline & 6 & & & & \\
\hline & 7 & & & & \\
\hline & 8 & & & & \\
\hline Total & & $18 \times 2$ & $18 \times 3$ & $16 \times 4$ & 154 \\
\hline
\end{tabular}

Fonte: autores.

Tabela 4 -

Roadmap de gestão educacional em desempenho administrativo

\begin{tabular}{|c|c|c|c|c|c|c|c|}
\hline Perspectiva & Indicador & 1 & 2 & 3 & 4 & 5 & Total \\
\hline \multirow{8}{*}{ 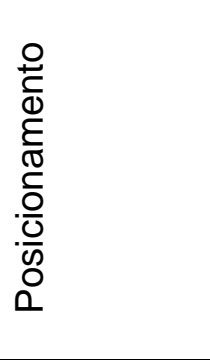 } & 1 & & & & & & \\
\hline & 2 & & & & & & \\
\hline & 3 & & & & & & \\
\hline & 4 & & & & & & \\
\hline & 5 & & & & & & \\
\hline & 6 & & & & & & \\
\hline & 7 & & & & & & \\
\hline & 8 & & & & & & \\
\hline \multirow{8}{*}{ 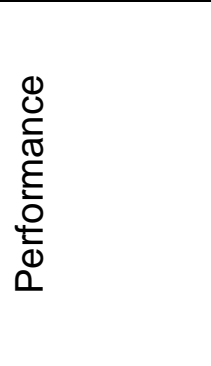 } & 1 & & & & & & \\
\hline & 2 & & & & & & \\
\hline & 3 & & & & & & \\
\hline & 4 & & & & & & \\
\hline & 5 & & & & & & \\
\hline & 6 & & & & & & \\
\hline & 7 & & & & & & \\
\hline & 8 & & & & & & \\
\hline Total & & & $3 \times 2$ & $8 \times 3$ & $5 \times 4$ & & 50 \\
\hline
\end{tabular}

Fonte: autores.

A figura 2 apresenta o mapa estratégico do RGE. A utilização das cores possibilita destacar os pontos fracos e fortes do que já foi mapeado, facilitando o estabelecimento de estratégias de melhorias com base no nível de maturidade e nos pontos críticos apresentado por cada um dos indicadores. O campo em verde significa que naquele 
indicador a unidade de ensino está num nível de maturidade desejado. Já o campo em amarelo significa que naquele indicador a unidade de ensino está num nível de maturidade que requer atenção. $O$ campo vermelho significa que naquele indicador a unidade de ensino está em nível de maturidade indesejado.

Figura 2 -

Roadmap de gestão educacional.

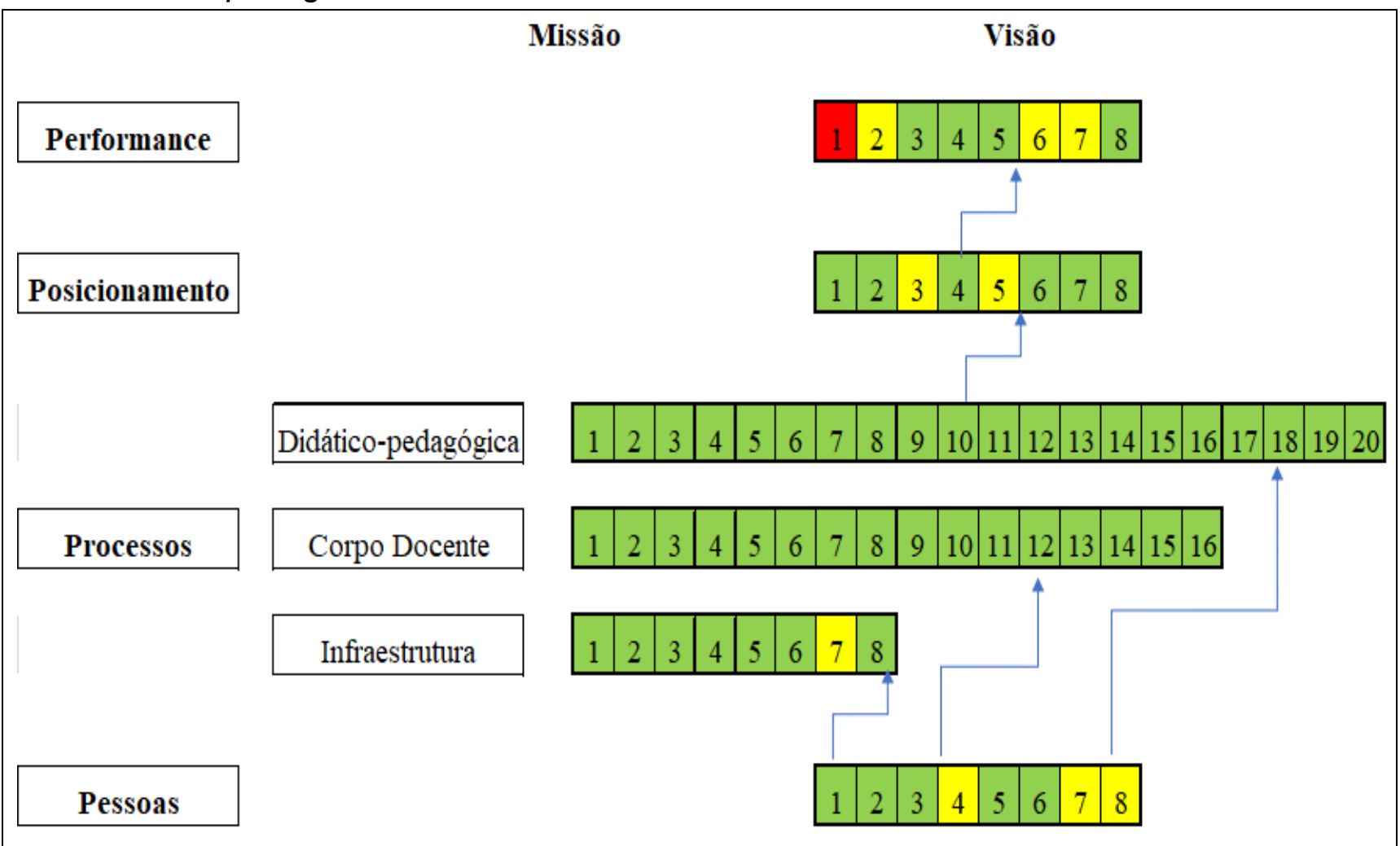

Fonte: autores.

\section{Perspectivas e indicadores do roadmap de gestão educacional}

O modelo foi organizado em quatro perspectivas: Performance, Posicionamento, Processos e Pessoas e em duas dimensões: Acadêmica e Desempenho.

Pessoas e Processos compõem a dimensão acadêmica do RGE, sendo que a primeira está baseada em inovação e aprendizado de Kaplan e Norton (1997). Já a segunda é baseada em três bases do Sinaes (Brasil, 2017): organização didáticopedagógica, corpo docente e infraestrutura. Estes indicadores poderão variar em virtude da área a qual está sendo avaliada, pois cursos na área da saúde e na área da educação, por exemplo, possuem particularidades específicas que devem ser respeitadas.

Posicionamento e Performance compõem a dimensão Desempenho do RGE e estão baseadas em Kaplan e Norton (1997), contudo, consideram a especificidade sustentabilidade do setor educacional. Na dimensão Acadêmica serão apresentados quatro quadros, cada qual com seus indicadores. O quadro 2 apresenta aqueles relacionados com a perspectiva Processos, com base em organização didáticopedagógica do Sinaes. 
Quadro 2 -

Processo acadêmico: organização didático-pedagógica.

\begin{tabular}{|c|c|}
\hline \multicolumn{2}{|r|}{ Mapa estratégico: perspectiva Processos (organização didático-pedagógica) } \\
\hline \multirow{20}{*}{ 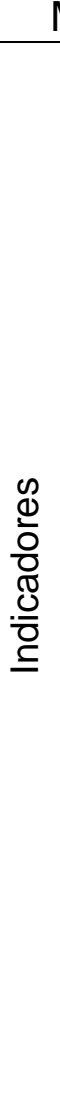 } & 1) Políticas institucionais do curso \\
\hline & 2) Objetivos do curso \\
\hline & 3) Perfil profissional do egresso \\
\hline & 4) Estrutura curricular \\
\hline & 5) Conteúdos curriculares \\
\hline & 6) Metodologia \\
\hline & 7) Estágio curricular supervisionado \\
\hline & 8) Atividades complementares \\
\hline & 9) Trabalho de conclusão de curso \\
\hline & 10) Apoio ao discente \\
\hline & 11) Processos de avaliação do curso \\
\hline & 12) Atividades de tutoria \\
\hline & 13) Tecnologias de informação e comunicação \\
\hline & 14) Material didático institucional \\
\hline & 15) Mecanismos de interação entre docentes, tutores e estudantes \\
\hline & $\begin{array}{l}\text { 16) Procedimentos de acompanhamento e de avaliação do ensino- } \\
\text { aprendizagem }\end{array}$ \\
\hline & 17) Número de vagas para o curso \\
\hline & 18) Integração com as redes públicas de ensino \\
\hline & 19) Integração do curso com o sistema local e regional de saúde/ SUS \\
\hline & 20) Atividades práticas de ensino \\
\hline
\end{tabular}
Fonte: Brasil (2017).

O quadro 3 apresenta os indicadores da dimensão acadêmica do RGE relacionados com a perspectiva Corpo docente. Eles foram selecionados com base no instrumento de avaliação do MEC para instituições de ensino superior (2018).

Quadro 3 -

Processo acadêmico: corpo docente.

\begin{tabular}{|c|c|}
\hline \multirow{14}{*}{ 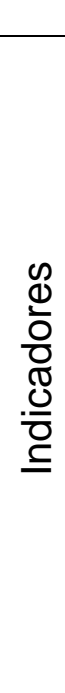 } & Mapa estratégico: Processos (Corpo Docente) \\
\hline & 1) Composição do NDE \\
\hline & 2) Qualificação da equipe multidisciplinar para a educação a distância \\
\hline & 3) Atuação do coordenador \\
\hline & 4) Regime de trabalho do coordenador \\
\hline & 5) Conteúdos dos componentes curriculares \\
\hline & 6) Regime de trabalho do corpo docente \\
\hline & 7) Experiência profissional do corpo docente \\
\hline & 8) Experiência acadêmica do corpo docente \\
\hline & 9) Experiência dos docentes em EAD \\
\hline & 10) Experiência do corpo tutorial \\
\hline & 11) Atuação do Colegiado \\
\hline & 12) Titulação dos Tutores \\
\hline & 13) Experiências do corpo de tutores \\
\hline
\end{tabular}


\begin{tabular}{|l|l|} 
14) Interação entre tutores, docentes e coordenador \\
\hline 15) Produção acadêmica de docentes nos últimos 3 anos. \\
\hline 16) Tecnologias de informação e comunicação
\end{tabular} Fonte: Brasil (2017).

O quadro 4 apresenta os indicadores da dimensão acadêmica do RGE relacionados com a perspectiva Infraestrutura, selecionados com base no instrumento de avaliação do MEC para instituições de ensino superior (2018).

Quadro 4 -

Processo Acadêmico Infraestrutura.

\begin{tabular}{|c|c|}
\hline \multirow{9}{*}{ 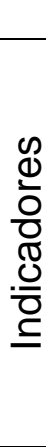 } & Mapa estratégico: Infraestrutura \\
\hline & 1) Espaços de trabalho para docentes \\
\hline & 2) Espaço de trabalho do coordenador \\
\hline & 3) Sala coletiva dos professores \\
\hline & 4) Salas de aula \\
\hline & 5) Laboratório de informática \\
\hline & 6) Requisitos relacionados a bibliografia básica \\
\hline & 7) Requisitos relacionados a bibliografia complementar \\
\hline & 8) Comitê de Ética em Pesquisa \\
\hline
\end{tabular}

Fonte: Brasil (2017).

O quadro 5 apresenta os indicadores da dimensão acadêmica do RG sob a perspectiva Pessoas. Tais dados foram selecionados com base nos itens inovação e aprendizado do BSC direcionada às instituições de ensino superior.

Quadro 5 -

Perspectiva Pessoas.

\begin{tabular}{|c|c|}
\hline \multicolumn{2}{|r|}{ Mapa estratégico: Pessoas } \\
\hline \multirow{8}{*}{ 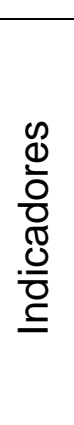 } & 1) \% de professores com desempenho excelente na avaliação discente \\
\hline & 2) \% de professores titulados \\
\hline & 3) Número de horas de formação dos professores \\
\hline & 4) Número de professores participando de eventos científicos \\
\hline & 5) Número de professores publicando em periódicos/eventos científicos \\
\hline & 6) Número de disciplinas realizadas fora da sala de aula convencional \\
\hline & 7) Números de projetos de pesquisa dos professores \\
\hline & 8) Números de projetos de extensão dos professores \\
\hline
\end{tabular}

Fonte: autores.

Na dimensão Desempenho do RGE serão apresentados dois quadros, cada qual com seus indicadores. O quadro 6 apresenta aqueles relacionados com a perspectiva Posicionamento, os quais foram selecionados com base no item clientes do BSC direcionada às instituições de ensino superior. Como foram escolhidos oito indicadores para este processo a avalição da unidade de ensino ficará compreendida entre 0 e 40 pontos, visto que cada um terá uma ponderação de 1 a 5 . 
Quadro 6 -

Perspectiva Posicionamento.

\begin{tabular}{|c|c|}
\hline \multirow{9}{*}{ 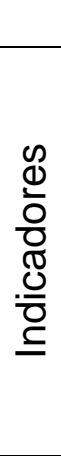 } & Mapa estratégico: Posicionamento \\
\hline & 1) \% Crescimento do número de alunos \\
\hline & 2) \% Alunos satisfeitos com a IES \\
\hline & 3) \% Alunos satisfeitos com corpo docente \\
\hline & 4) $\%$ Alunos matriculados \\
\hline & 5) \% Redução de alunos evadidos \\
\hline & 6) \% Crescimento de alunos ingressantes \\
\hline & 7) \% Crescimento de alunos em estágio \\
\hline & 8) Escore da IES no Enade \\
\hline
\end{tabular}

Fonte: autores.

O quadro 7 do RGE apresenta os indicadores da dimensão desempenho, referentes à perspectiva Performance, os quais foram selecionados com base nos pontos financeiros do BSC direcionada às IES. Como foram escolhidos oito indicadores para este processo e a avalição da unidade de ensino ficará compreendida entre 0 e 40 pontos, visto que cada indicador terá uma ponderação de 1 a 5 .

Finalmente, como os autores Kaplan e Norton (2004), em seu trabalho de divulgação do BSC, defendem possibilidade de incorporar novas perspectivas, neste estudo foi adicionada indicadores sociais na perspectiva Performance, em razão de sua importância dessa dimensão dentro do setor educacional.

Quadro 7 -

Perspectiva performance.

\begin{tabular}{|c|c|}
\hline \multirow{9}{*}{ 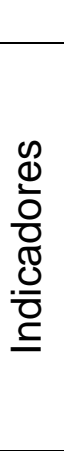 } & Mapa estratégico: performance \\
\hline & 1) $\%$ Crescimento da rentabilidade \\
\hline & 2) \% Crescimento do faturamento \\
\hline & 3) \% Crescimento resultado operacional \\
\hline & 4) \% Redução de custos \\
\hline & 5) \% Crescimento da participação de mercado \\
\hline & 6) Número de projetos sociais \\
\hline & 7) Número de projetos de extensão \\
\hline & 8) Número de projetos de pesquisa \\
\hline
\end{tabular}

Fonte: Sinaes (2018).

\section{Conclusão}

O RGE é capaz de categorizar os cursos em relação aos requisitos do Sinaes, possibilitando o desenvolvimento de políticas públicas mais eficientes para redes de ensino superior. Assim sendo, torna-se possível ter um diagnóstico dos cursos de uma IES, ou ainda dos cursos de uma rede de ensino como um todo.

De fato, o RGE permite a elaboração de estratégias de melhorias de processos acadêmicos e administrativos em IES, havendo a possibilidade de verificar pontos de melhoria comuns em redes de ensino superior públicas ou privadas, visando a orientar esforços coletivos de aprimoramento de processos acadêmicos e administrativos. Nesse 
sentido há, portanto, a possibilidade de se utilizar o RGE como um instrumento de diagnóstico de IES para definição de políticas públicas de ensino superior.

O RGE, após sua validação em contextos empíricos, poderá ser um instrumento de gestão estratégico de IES de caráter diferenciado na gestão educacional por ter a capacidade de medir as dimensões acadêmica e administrativa e ainda correlacioná-las no intuito de compreender as relações entre baixo/alto desempenho administrativo versus baixo/alto desempenho acadêmico.

Por fim, acredita-se que o RGE é um modelo de maturidade adequado para gestão educacional de IES e pode ser desenvolvido de forma ampla em seu campo de estudo, por se tratar de um instrumento estratégico que tem a capacidade de auxiliar no processo de gestão educacional.

\section{Referências}

ALACH, Zhivan. Performance measurement maturity in a national set of universities. International Journal of Productivity and Performance Management, Bingley, West Yorkshire, England, v. 66, n. 2, 2017, pp. 216-230.

ALBOUSHRA, Marwa; SHAHBUDIN, Shah Md Shahbudin; ABDALLA, Yousif. Understanding challenges of performance measurement in a public university: evidence from Sudan. Asian Social Science, Richmond Hill, Ontario, Canada, v. 11, n. 15, 2015, p. 10.

AZMA, Fereydoon. Qualitative indicators for the evaluation of universities performance. Procedia Social and Behavioral Sciences, Amsterdam, Netherlands, v. 2, n. 2, 2010, p. 5408-5411.

BEARD, Deborah. Successful applications of the balanced scorecard in higher education. Journal of Education for Business, Newark, Delaware, v. 84, n. 5, 2009, p. 275-282.

BHAGWAT, Rajat; SHARMA, Milind Kumar. Performance measurement of supply chain management: A balanced scorecard approach. Computers \& Industrial Engineering, Los Angeles, California, v. 53, n. 1, 2007, p. 43-62.

BRASIL. Sistema nacional de avaliação da educação superior Sinaes: instrumento de avaliação de cursos de graduação presencial e a distância. Brasília: MEC, 2017.

CHEN, Shun-Hsing; YANG, Ching-Chow; SHIAU, Jiun-Yan. The application of balanced scorecard in the performance evaluation of higher education. The TQM magazine, Bingley, West Yorkshire, England, v. 18, n. 2, 2006, p. 190-205.

CULLEN, John; JOYCE, John; HASSALL, Trevor Hassall; BROADBENT, Mick. Quality in higher education: from monitoring to management. Quality Assurance in Education, Sheffield, v. 11, n. 1, 2003, p. 5-14.

DE ANDRADE, José Baltazar Salgueirinho Osório et al. A proposal of a balanced scorecard for an environmental education program at universities. Journal of Cleaner Production, New Rochelle, NY, USA, v. 172, 2018, p. 1674-1690.

DE BIAZZI, Monica Rottmann de; MUSCAT, Antonio Rafael Namur; DE BIAZZI, Jorge Luiz de. Modelo de aperfeiçoamento de processos em instituições públicas de ensino superior. Gestão e Produção, São Carlos, v. 18, n. 4, 2011, p. 869-880.

DINÇER, Hasan; YÜKSEL, Serhat; MARTÍNEZ, Luis. balanced scorecard-based analysis about european energy investment policies: a hybrid hesitant fuzzy decision-making approach with quality function deployment. expert systems with applications, Expert 
Systems with Applications, Shreveport, Louisiana, United States of America, v. 115, 2019, p. $152-171$.

FISHER, David. The business process maturity model: a practical approach for identifying opportunities for optimization. Business Process Trends, Newton, MA, USA v. 9, 2004, p. 11-15.

HAMMER, Michael. The process audit. Harvard Business Review, Boston, MA, USA. 2007, p.111-123.

HLADCHENKO, Myroslava. Balanced scorecard-a strategic management system of the higher education institution. International Journal of Educational Management, Bingley, West Yorkshire, England, v. 29, n. 2, 2015, p. 167-176.

KALAKOTA, Ravi; ROBINSON, Marcia. E-business 2.0: roadmap for success. Boston, MA, USA: Addison-Wesley Longman Publishing, 2001.

KAPLAN, Robert Samuel; NORTON, David. The balanced scorecard: measures that drive performance, Harvard Business Review, Boston, MA, USA, 1992, p. 71-79.

KAPLAN, Robert Samuel; NORTON, David. A estratégia em ação: balanced scorecard. Rio de Janeiro: Campus. 1997.

KAPLAN, Robert Samuel; NORTON, David. Mapas estratégicos-balanced scorecard: convertendo ativos tangíveis em resultados tangíveis. Rio de Janeiro: Campus, 2004.

LAWRENCE, Stewart; SHARMA, Umesh. Commodification of education and academic labour: using the balanced scorecard in a university setting. Critical perspectives on accounting, Sydney, Australia, v. 13, n. 5-6, 2002, p. 661-677.

LEE, Jihyun; LEE, Danhyung; LEE, Sungwon Kang. An overview of the business process maturity model (BPMM). International Workshop on Process Aware Information Systems, New York City: Springer, 2007.

LIPE, Marlys Gascho; SALTERIO, Steven. The balanced scorecard: Judgmental effects of common and unique performance measures. The Accounting Review, Lakewood Ranch, FL, USA, v. 75, n. 3, 2000, p. 283-298.

LOCKAMY, Archie; MCCORMACK, Kevin. The development of a supply chain management process maturity model using the concepts of business process orientation. Supply Chain Management, Bingley, West Yorkshire, England, v. 9, n. 4, 2004, p. 272278.

MAULL, Roger; TRANFIELD, David; MAULL, Willian. Factors characterizing the maturity of BPR programs. International Journal of Operations \& Production Management, Bingley, West Yorkshire, England, v. 23, No. 6, 2003, p. 596-624.

NAYERI, Mahmoud Dehghan; MASHHADI, Mahmoud Mosavi; MOHAJERI, Kaveh. Universities strategic evaluation using balanced scorecard. World Academy of Science, Engineering and Technology, Barcelona, v. 37, n. 1, 2008, p. 332-334.

NICKEL, Sigrun. Strategic management in higher education institutions-approaches, processes and tools. Leadership and Governance in Higher Education, Hoboken, New Jersey, USA, v. 3, 2011, p. 23.

PMI. Organizational project management maturity model (OPM3). Newtown Square, PA, USA: Project Management Institute, 2003.

RODHOLFF, Michael. Case study and maturity model for business process management implementation. LNCS, Berlin, v. 5701, 2009, p. 128-142. 
RÖGLINGER, Maximilian; PÖPPELBUB, Jens; BECKER, Jörg. Maturity models in business process management. Business Process Management Journal, Augsburg, v. 18, 2012, p. 328-346.

ROSEMANN, Michael; DE BRUIN, Tonia. Towards a business process management maturity model. EUROPEAN CONFERENCE ON INFORMATION SYSTEMS, 2005. Anais ... Regensburg: Ecis, 2005.

$\mathrm{YU}$, May Leen et al. The e-balanced scorecard (e-BSC) for measuring academic staff performance excellence. Higher Education, Lansing, MI, USA, v. 57, n. 6, 2009, p. 813828.

Geraldo Magela Jardim Barra é professor na Universidade Federal de São João del-Rei. Orcid: https://orcid.org/0000-0001-7186-6344.

Endereço: Campus Ctan - Avenida Visconde do Rio Preto, s/n - Colônia do Bengo, Prédio Direito - 36300-000 - São João del-Rei - MG - Brasil.

E-mail: gmjbar@yahoo.com.br.

Rodrigo Oliveira da Silva é professor na Faculdade de Administração e Ciências Contábeis da Universidade Federal de Juiz de Fora.

Orcid: https://orcid.org/0000-0001-7936-3418.

Endereço: Rua José Lourenço Kelmer, s/n - São Pedro - 36036-900 - Juiz de Fora MG - Brasil.

E-mail: oliveira.silva@ufjf.edu.br.

Marilia Medeiros Schocair é estudante do curso de doutorado em Administração na Unisinos.

Orcid: https://orcid.org/0000-0002-0727-2311.

Endereço: Rua Embaixador Assis Chateaubriand, 58/206 - Volta Redonda - RJ Brasil.

E-mail: marilia.schocair@gmail.com.

Critérios de autoria: todos os autores participaram da concepção da ideia e problema de investigação da pesquisa, assim como da análise dos dados e redação do texto. Geraldo elaborou o quadro de referência e modelo cerne da pesquisa. Rodrigo executou a busca de literatura que ampara as reflexões sobre o problema de investigação. Marília apoiou a análise e desenvolvimento da metodologia que balizou o estudo.

Recebido em 13 de janeiro de 2021.

Aceito em 19 de maio de 2021.

(c) (i) $(\$$ 